\title{
¿ES CORRECTO INVESTIGAR CON SERES HUMANOS?
}

Paula Prieto Martínez MD*

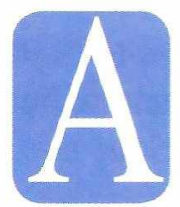

lo largo de la historia de occidente, los médicos y científicos han querido saber más acerca de la fisiología y la patología del ser humano y han usado diferentes métodos para obtener dicha información en aras de mejorar la salud de los hombres y por consiguiente su calidad de vida. Aunque no siempre fue una investigación formal como ahora la conocemos, se realizaban actos unidos a la práctica clínica que aumentaban los conocimientos científicos. Sin embargo, como lo veremos más adelante, existen otras motivaciones diferentes a la de la búsqueda de tratamientos novedosos para beneficiar a los pacientes, que hacen necesaria la reflexión sobre la pertinencia de "usar" sujetos en estos procesos de investigación.

El objetivo de este artículo es hacer un recuento histórico de lo que ha sido la investigación con seres humanos y tratar de argumentar esta práctica desde el punto de vista ético, ya que involucra la participación de sujetos que en el proceso pueden convertirse en "objetos". La pregunta que planteo para desarrollar lo anterior es: ¿Es ético investigar con seres humanos? Mi propuesta es que en la medida que la investigación se haga con objetivos claros y benéficos para los hombres que participan y para la humanidad, realizándose con sujetos que consienten participar de forma autónoma, se puede justificar éticamente su realización.

Empezaré haciendo unas aclaraciones de términos, luego unas apreciaciones históricas que revelan como ha sido la investigación a lo largo de los siglos, para luego justificar éticamente la investigación de acuerdo con unos parámetros específicos, haciendo

Médica especialista en bioética de la Universidad Javeriana, Instructora Asistente Div. de nvestigaciones y de la Div. de Bioética de la Fundación Universitaria de Ciencias de la Salud. énfasis en el papel que juegan los comités de ética en la supervisión de este proceso.

De acuerdo con Diego Gracia, es necesario aclarar los conceptos mismos implicados en estas actividades, para después hacer un recuento histórico de las etapas que se han delimitado a lo largo de la misma. ${ }^{1}$ Para el autor, por clínica se entiende como todas las acciones que se realizan sobre el cuerpo humano sean sujetos sanos o enfermos, para conocer y tratar mejor las enfermedades. Esta actividad puede ser de dos tipos: la práctica clínica y la investigación clínica. La primera es todo el acto realizado en el cuerpo de un paciente para diagnosticar y tratar una enfermedad, mientras que la investigación está encaminada a conocer el diagnóstico o las aplicaciones terapéuticas. La investigación implica una actividad designada a comprobar una hipótesis, sacar conclusiones para obtener conocimientos generalizables (o la llamada validez externa).

El mismo autor divide la historia de la ética de la investigación en tres períodos definidos: la primera desde los inicios de la medicina hasta finales del siglo XIX. La segunda desde principios del siglo veinte hasta el año 1947 y la última desde ese año hasta el 2000. Sin embargo, algunos autores latinoamericanos como la argentina Silvia Brussino, incluyen una última etapa a partir del 2000 hasta nuestros días, denominada post-Helsinki.

\section{Primer período (-- a 1900)}

En la primera etapa, la clínica clásica, el efecto principal buscado en el acto médico era curar o aliviar al enfermo, o sea la intención era con fines benéficos, mientras que la investigación clínica era toda la actividad realizada en una persona enferma con intención cognoscitiva. Se establece un principio de doble efecto, pilar de la ética clásica, donde ciertos efectos 
indeseables que se derivan de un acto necesario, realizado siempre con intención benéfica, son moralmente justificados. Por consiguiente, el efecto principal que se buscaba con el acto médico era curar o aliviar al enfermo, pero durante el mismo, por doble efecto, el médico podía adquirir conocimientos.

Para obtener conocimientos sobre la fisiología y patologías humanas, los procedimientos permitidos eran: la analogía, o sea aplicar conocimientos al ser humano que se habían adquirido en otras especies biológicas; el azar, ya que las heridas ocasionadas en guerras o accidentes servían de aprendizaje a los médicos que trataban de curarlas y por último la enfermedad misma, como situación experimental de la que el médico aprendía mientras trataba de diagnosticarla y curarla. La investigación con sólo fines cognoscitivos estaba permitida en animales, cadáveres y en condenados a muerte, que generaba controversia en especial en lo referente a la vivisección en seres humanos, por ser actos crueles e inhumanos. Aunque fueron prácticas frecuentes, no había consenso en la comunidad médica por considerarlas inmorales ya que distorsionaba los fines propios de la medicina. Un ejemplo de este tipo de investigación fue realizado por el médico William Beaumont(1785-1853) en un paciente a quien una herida había dejado expuesto el estómago, lo que permitió al médico observar la fisiología gástrica mediante un tipo de contrato con el paciente que participó en forma voluntaria en este experimento.

Sin embargo, los cambios en la lógica y en la forma de acceder al conocimiento a partir del siglo XVII, permitieron el desarrollo del método experimental en el ejercicio de la medicina como evidencian las respuestas de Thomas Percival (finales del siglo XVIII) y de Claude Bernard (mediados del siglo XIX) cuando se evidenció la necesidad de experimentar pero sin perder de vista el beneficio del paciente. A pesar de esta premisa, se llevaron a cabo numerosos experimentos farmacológicos y quirúrgicos donde pareció perderse la intención terapéutica frente a la obtención de conocimiento útil. Percival no condenó el avance de la ciencia que se perfilaba en la medicina, pero recomendó un proceder guiado por la sana razón o sea una recta conciencia. ${ }^{2}$

\section{Segundo período (1900-1947)}

Aunque se realizaban experimentos en la época anterior, la intención primordial era buscar el beneficio del paciente, sin tener en cuenta el consentimiento de los sujetos, ya fueran enfermos o sanos. Esto se explica por el modelo paternalista de ejercicio de la profesión médica que se mantuvo vigente hasta mediados del siglo XX. Se suponía que el médico sabía lo que era bueno para el paciente.

Sin embargo, en el campo de la experimentación tanto en Europa como en los Estados Unidos, ya no estaba tan claro que el beneficio de un paciente concreto fuera el criterio único y fundamental para justificar la experimentación. Esto sumado al cambio en la mentalidad sobre la investigación empírica, hizo que se impusiera una tesis exactamente contraria a la que había imperado en siglos anteriores: el beneficio real para los pacientes no estaba solo en la intención del médico de hacer el bien, sino que el beneficio tenía que estar basado en pruebas que solo se podían obtener por la investigación experimental.

Luego se pasa, mediante la investigación clínica, de una medicina basada en la "intención" a una medicina basada en la "evidencia". La validación o investigación clínica con seres humanos tenía que ser posible por ella misma y no por accidente. Este cambio en la lógica de la investigación clínica se debió principalmente al cambio de la lógica de la investigación científica, que se introdujo en el ámbito de las ciencias biológicas en las primeras décadas del siglo XX. Ante la crisis del conocimiento empírico, al hacerse evidente que las proposiciones de carácter universal, como son las científicas, no podían ser más que probables, y con la aparición de la estadística como método natural de la racionalidad científica, se hizo imperativo verificar su carácter probable mediante continua revisión. Existieron otras razones para el inicio de esta etapa además de la convicción de que el conocimiento empírico tenía carácter necesariamente estadístico, como son la insuficiencia del principio de analogía y la necesidad de controlar el aprendizaje meramente fortuito. 
La tesis clásica de que los fenómenos fortuitos de la vida como el azar y la enfermedad colocaban al médico ante claras situaciones experimentales, razón por la cual no era necesario provocarlas en forma artificial, no era suficiente para el manejo de algo tan importante como la salud y por lo tanto fue necesario introducir una idea nueva: la del diseño experimental, iniciando una etapa de experimentación con seres humanos con la elaboración sistemática de los grandes principios de la estadística descriptiva y muestral ${ }^{1}$.

Se llevaron a cabo durante las tres primeras décadas del siglo XX numerosos experimentos clínicos con seres humanos que consentían participar pero los abusos estaban presentes por los riesgos que implicaban, ya que se justificaban experimentos que causaban daños inequívocos, incluso la muerte, en la medida que los sujetos hubieran dado su consentimiento voluntario. Esto dio origen a una nueva ética ya que el beneficio para el paciente no era el fin último y no estaba tan claro que la investigación fuera beneficiosa para un paciente concreto. Apareció entonces el concepto de respeto a la autonomía de los sujetos de experimentación, haciendo que pruebas aún riesgosas para la vida de los sujetos, fueran válidas si se tenía su consentimiento. Se presentó un adelanto en el campo de la autonomía del paciente pero un retroceso en la beneficencia. Sin embargo, los muchos abusos cometidos pusieron en tela de juicio el avance de la autonomía cuando las investigaciones se llevaban a cabo, en la mayoría de los casos, en hospitales de beneficencia, con pacientes pobres o marginales, con escasa instrucción y sin evaluar de manera adecuada los riesgos frente a los beneficios. ${ }^{2}$

Ante esta situación surge la necesidad de una regulación legal de la experimentación. Ya en el año 1931 en Alemania se promulga una ley que da importancia fundamental al respeto de la autonomía y en 1935 en los Estados Unidos se permitía una experimentación con seres humanos si daban su consentimiento. ${ }^{1}$ Durante la Segunda Guerra Mundial, los investigadores alemanes, muchos de ellos médicos, realizaron numerosos procedimientos en campos de concentración con poblaciones obviamente vulnerables, en especial prisioneros judíos y gitanos, pero también prisioneros políticos y otros. Algunos de los objetivos de estas investigaciones eran los de encontrar la forma más eficiente y rápida de esterilizar, que incluía radiaciones clandestinas y la castración sin anestesia tanto masculina como femenina, o técnicas para producir la muerte de manera indetectable, de forma individual o masiva. Dentro de las experimentaciones más conocidas, están aquellas sobre hipotermia, donde se probaban mecanismos para producir la muerte por congelamiento y así lograr evitarla en sus soldados durante el invierno, sobre todo en los caídos en el Mar del Norte, y lo hacían sumergiendo a los prisioneros en agua helada y observando los cambios fisiológicos que se producían hasta la muerte.

Paralelo a los estudios practicados por los nazis, en Japón se llevaron a cabo experimentos con armas biológicas en al menos once ciudades chinas, además de provocar gangrena gaseosa en tres mil prisioneros de guerra al explotar bombas cerca de sus extremidades expuestas en un centro de detención llamado Unidad $731 .^{3}$

Dado el respeto a la autonomía planteado en este periodo, así como la autonomía dada a los investigadores, confiando en su capacidad moral para la realización de las investigaciones considerando innecesario e inconveniente legislar sobre estas situaciones, no sorprende lo escandaloso y aterrador que fueron los testimonios sobre estas atrocidades cometidas durante la Segunda Guerra Mundial, llevadas a cabo muchas de ellas por científicos reconocidos.

Las experiencias anteriores sentaron las bases para la promulgación del Código de Nüremberg, primer documento internacional regulatorio sobre la experimentación en seres humanos con que culmina la ética de este segundo período. El documento, cuyo objetivo es proteger la integridad de la persona que se somete a un experimento, estipula las condiciones necesarias para la realización de trabajos de investigación con seres humanos, poniendo especial énfasis en el consentimiento voluntario de las personas participantes de los estudios. 
Fue un código orientador para los investigadores quienes a pesar de las atrocidades cometidas, seguían pensando que no era necesaria una reglamentación más específica. Por el contrario, se les debía pedir a los investigadores autocontrol y una elevada condición moral. (1) Así lo expuso Beecher en 1959 al publicar un libro llamado Experimentation in Man, donde planteaba la necesidad no solo de considerar el consentimiento informado sino de hacer una diferencia clara entre la práctica clínica y la de investigación. En la primera se podía buscar el beneficio del paciente continuando con una práctica paternalista y en la segunda insistía en la autorregulación de los científicos.

\section{Tercer período (1947-1999)}

Lo vivido durante la Segunda Guerra Mundial fue muestra evidente para demostrar que la autorregulación por parte de los científicos no era suficiente para realizar una investigación con seres humanos de forma correcta.

Por su parte, la Declaración Universal de los Derechos Humanos, adoptada por la Asamblea General de las Naciones Unidas en 1948, y el Acuerdo Internacional sobre Derechos Civiles y Políticos de 1966, estipulan que: "Nadie será sometido a tortura o a un tratamiento o castigo cruel, inhumano o degradante. En especial, nadie será sometido sin su libre consentimiento a experimentación médica o científica".

A pesar de lo anterior y de la promulgación del Código de Nüremberg, las injusticias continuaron como lo publicó Beecher en 1966 en un artículo en el New England Journal of Medicine, Ethics and Clinical Research donde denunciaba investigaciones concretas con serios problemas éticos e insistía en el juicio correcto y virtuoso de los científicos más que en las reglamentaciones rígidas. Sus comentarios influyeron y sensibilizaron al público norteamericano y esto aumentó en forma considerable al darse a conocer a la opinión publica otras arbitrariedades cometidas, como el caso del Hospital Judío de Enfermedades Crónicas en Brooklin, Nueva York, en 1963, donde se inyectaron por vía subcutánea células cancerosas a 22 pacientes ancianos sin su consentimiento para conocer la respuesta inmunológica; o el ocurrido en la Escuela Estatal de Willowbrook para niños, muchos de ellos con discapacidad mental, en Staten Island, Nueva York, donde con el fin de buscar una vacuna contra la hepatitis un grupo de médicos inició en 1956 un estudio infectando deliberadamente de hepatitis a los niños recién ingresados, diciendo que de todas maneras se infectarían al estar en el centro por el hacinamiento en que se encontraban y que sólo seguirían el curso natural de la enfermedad. Al parecer los padres firmaron un consentimiento informado pero bajo la presión de obtener un cupo si permitían que sus hijos participaran en el estudio. También fue denunciado por Beecher en 1970.

Otras investigaciones fueron realizadas con las mismas violaciones ya descritas como el realizado en Nueva Zelanda en 1966 con mujeres, cuando se hizo un seguimiento del cáncer de cuello uterino in situ durante diez años sin recibir el tratamiento adecuado, sabiendo que se podía evitar su progresión con medidas conocidas. Otras investigaciones no éticas fueron conducidas por los militares norteamericanos durante y después de la Segunda Guerra Mundial, en las cuales soldados en servicio fueron expuestos en forma no voluntaria a agentes químicos como gas mostaza; o estudios apoyados por el mismo gobierno donde 18 pacientes terminales recibieron radiaciones con plutonio entre 1945 y 1947 o en una institución de incapacitados mentales en Massachussets, donde jóvenes recibieron pequeñas cantidades de hierro y calcio radioactivo para observar el proceso de absorción en el cuerpo. ${ }^{3}$

En 1964, la Asociación Médica Mundial, en Helsinki (Beecher fue su principal redactor), promulgó la Declaración de Helsinki en la que aparecían en forma clara dos capítulos, uno dedicado a la investigación médica combinada con asistencia profesional y el otro referente a la investigación biomédica no terapéutica. Esta declaración ha tenido considerable influencia en la formulación de las legislaciones y de los códigos de conducta internacionales, regionales y nacionales. Fue enmendada por la $29^{\mathrm{a}}$ Asamblea en Tokio, en 1975, y revisada de nuevo en la $35^{\mathrm{a}}$ 
Asamblea en Venecia, en 1983. También sufrió revisiones en la $41^{\text {a }}$ Asamblea de Hong Kong, en 1989, en la 48 Asamblea General en Somerset West, Sudáfrica, en 1996 y en la 52a Asamblea General en Edimburgo, Escocia, en el año 2000.

Se trata de un planteamiento internacional de gran alcance. Fundamentalmente, establece pautas éticas para los médicos que realizan investigación biomédica clínica y no clínica, y estipula, entre sus diversas reglas, el consentimiento informado de las personas que participan en la investigación. Adaptó sus principios a la ética de la profesión médica e introdujo las nociones de riesgo- beneficio para los sujetos experimentales y el estudio de los protocolos de investigación por un comité independiente de los investigadores, lo que ha sido mantenido y reforzado en la última revisión.

A principios de 1932 el Servicio de Salud Pública de los Estados Unidos inició un estudio para seguir el curso de la sífilis sin tratamiento en una población negra, incluyendo a cuatrocientos hombres de raza negra con diagnóstico de sífilis en comparación con otros doscientos sin la enfermedad. El estudio, cuya primera publicación científica apareció en 1936 y otras doce veces en revistas médicas prestigiosas fue revisado varias veces entre 1932 y 1970, y continuó hasta 1972 cuando fue denunciado en el New York Times, lo que llevó a su terminación.

Los participantes no fueron informados de manera correcta sobre la enfermedad, sobre el propósito del estudio, ni de los procedimientos a los que serían sometidos (como las punciones lumbares, las cuales, decían, eran parte del tratamiento) ni recibieron terapia adecuada aún cuando la penicilina estuvo disponible y se probó su efectividad para la patología estudiada. Además se bloqueó en forma sistemática cualquier intento de tratar a estos pacientes. En 1936 ya era evidente que las complicaciones eran mayores en los pacientes infectados que en el grupo de control y que el número de muertes era dos veces superior en los sifilíticos que en los sanos.
Se presentó además el escándalo de la talidomida en 1961 cuando Lenz en Alemania y McBride en Australia evidenciaron su efecto sobre el desarrollo de las extremidades en embriones humanos. Este medicamento se utilizó como antiemético y sedante en pacientes embarazadas durante el primer trimestre de gestación, sin tener conocimientos suficientes sobre los posibles efectos en los embriones. En 1963 la FDA hizo público un nuevo reglamento que había de regir la experimentación con nuevos fármacos y los NIH y el Departamento de Salud y Bienestar aplicaron criterios parecidos a toda la investigación biomédica y en 1966 hicieron públicas unas normas en las que obligaban que los protocolos fueran revisados por un comité de la institución. ${ }^{1}$

Se había establecido en la Declaración de Helsinki, y aquí se reitera, la importancia de la revisión de los protocolos de investigación por parte de un comité independiente. Ya no son suficientes el criterio y la autorregulación del investigador. Los puntos que se debían considerar eran: tener en cuenta los derechos y bienestar del paciente, el consentimiento informado y la relación riesgo / beneficio.

Ante lo expuesto antes y sobre todo después de conocerse lo sucedido en Tuskegee, el gobierno de los Estados Unidos creó en 1974 la Comisión Nacional para la Protección de los Sujetos Humanos de Investigación Biomédica y del Comportamiento, con el propósito de establecer la fundamentación ética que debía existir en toda investigación que involucrara seres humanos. En 1979 después de trabajar cuatro años, esta comisión produjo un documento conocido como el Informe Belmont. ${ }^{4}$ En él se establecen unos principios éticos que servirán de base para formular directrices específicas y cuyo objetivo primordial es proteger a los sujetos de investigación. Estos principios son: respeto a la persona, beneficencia y justicia.

El respeto a las personas, fundamentado en dos conceptos: todas las personas deben ser tratadas como seres autónomos y aquellas con autonomía disminuida tienen derecho a ser protegidas. Entendiendo autonomía como" la capacidad de deliberar sobre sus fines personales y de obrar bajo los fines de esta 
deliberación"4, por consiguiente hay que respetar las consideraciones y acciones de dichas personas a menos que sean claramente perjudiciales para los demás. Se entiende como beneficencia, toda acción que produzca bienestar en un sujeto y genera la obligación de no causar ningún daño. Esto implica determinar cuando buscar ciertos beneficios midiendo de forma estricta los riesgos que éstos conllevan. La justicia, entendida como la distribución equitativa tanto de las cargas como de los beneficios durante una investigación. Estos principios tienen una aplicación práctica en el campo de la investigación propiamente dicha.

Consentimiento informado entendido, no como un requisito obligatorio, sino como un proceso mediante el cual la persona recibiendo toda la información, con la comprensión adecuada y de forma en verdad voluntaria (sin coerción ni influencias indebidas) acepta participar en forma en una investigación. Valoración adecuada de los riesgos y beneficios, mediante un balance de la probabilidad de aparición de daños contra los posibles valores positivos para la salud o bienestar tanto del sujeto como de la comunidad, o sea" la valoración de riesgo/beneficio se refiere a las probabilidades y a las magnitudes de daños posibles y a los beneficios anticipados." Selección justa de los sujetos, tanto a nivel individual como social, teniendo especial cuidado con las poblaciones vulnerables. ${ }^{4}$

Después de este informe a lo largo de la historia se han publicado numerosas reglamentaciones a nivel internacional, enfocadas en especial a la investigación transnacional que se desarrolla en países en vía de desarrollo. Es así como en 1982 el Consejo de las Organizaciones Internacionales de Ciencias Médicas (sigla en inglés CIOMS), publica las Guías Éticas Internacionales para la Investigación Biomédica que involucra Seres Humanos, cuyo objetivo es ayudar a los investigadores e instituciones y a las autoridades sectoriales, regionales, nacionales e internacionales, a establecer y mantener normas para evaluación ética de los estudios epidemiológicos.

Se hace referencia sobre todo a la justificación ética y la validez científica de una investigación, al consentimiento informado, a la vulnerabilidad de los sujetos, grupos, comunidades y poblaciones, a la mujer como sujeto de investigación, a la igualdad en la distribución de las cargas y los beneficios ,a la confidencialidad, a las compensaciones en caso de efectos adversos, al fortalecimiento de los comités de revisión tanto locales como nacionales, a las obligaciones de los patrocinadores de la investigación haciendo énfasis en la protección de los sujetos y a su uso en los países que están definiendo sus propias pautas éticas. Después en 1998, se inició la revisión de estas Pautas Éticas, que sustituyen a las propuestas en 1982 y revisadas en el año 1993.

Por último, después de discutir diversos temas éticos que en las pautas no tenían disposiciones específicas, en especial aquellos que afectan a los países en desarrollo, y quedando algunos sin resolver, como el uso del placebo (Pauta 11), se editan las nuevas Pautas en marzo del 2000, puestas a disposición en el sitio Web de CIOMS en junio de 2001 y la siguiente versión en enero de 2002. La traducción a la lengua española estuvo a cargo de un grupo de profesionales convocados por el Programa Regional de Bioética de la Organización Panamericana de la Salud (Oficina Regional de la Organización Mundial de la Salud). El informe del Comité Asesor sobre experimentos humanos con radiaciones desarrolló en extenso el problema del engaño o la falsa información a sujetos experimentales. ${ }^{5}$

Así mismo, cada país ha implementado su propia reglamentación en aras de proteger a los sujetos de investigación, como en Colombia con la Resolución número 008430 de 1993, expedida por el entonces Ministerio de Salud: "por la cual se establecen las normas científicas, técnicas y administrativas para la investigación en salud."

\section{Cuarto período (2000 hasta el presente)}

Para algunos autores latinoamericanos como Silvia Brussino, Volnei Garrafa y Miguel Kottow se inicia una cuarta etapa que empieza a finales de los años ochenta cuando se extrema la tensión entre los derechos de las personas y los inocultables intereses de la industria farmacéutica y biotecnológica que podría llamarse modelo postHelsinki. 
Después de la última revisión de la Declaración de Helsinki en el año 2000 en Edimburgo se crea una controversia manifestada así por Kottow: "Esta última versión ha sido controvertida por su reprobación del uso de placebos y por requerir que los probandospacientes tengan acceso a los beneficios terapéuticos que emanen de la investigación en la cual participan y cuya disponibilidad debe continuar más allá de la compleción del estudio...Ambas exigencias han sido combatidas por los intereses de las instituciones patrocinantes y de los científicos, llevando a una situación conflictiva entre los intereses creados que sugieren un doble estándar ético que sea menos riguroso frente a las realidades de los países huéspedes de lo exigido en sus propias naciones, y a las protestas de los países más pobres que se resisten a ser tratados como campo de experimentación con una ética abreviada. Conflictos adicionales y aún no resueltos se generan por la explícita exigencia de la Declaración de Helsinki de cautelar al máximo los intereses y las necesidades de los pacientesprobandos en estudios clínicos tanto terapéuticos como no terapéuticos. Los investigadores prefieren distinguir entre ambas situaciones, estimando que la ética clínica es privativa para el paciente, en tanto que su incorporación a un estudio lo transforma en probando sujeto a una ética de investigación que no ha de cuidar los requerimientos terapéuticos sino la limpidez del protocolo científico."

Agrega el autor, además, "que la ética de investigación como cualquier otra codificación moral, debe cumplir tres requisitos: la no contradicción, el respeto por sus antecedentes histórico sociales y la consideración de su realidad social, quedando así inmune a la crítica externa que no tiene razón de aplicarse ya que proviene de otro ámbito cultural..........Diseñar una ética aspiracional para las investigaciones en países desarrollados es moralmente correcto pero no autoriza a modificar y empobrecerla si los mismos investigadores trasladan sus estudios a países menos desarrollados argumentando que las condiciones locales autorizarían una ética pragmática". ${ }^{8}$

En el año 2002, en Londres se publica la "Ética de Investigación Relativa a la Atención Sanitaria en los Países en Desarrollo" por el Consejo de Bioética de Nuffield, en la cual se revisa la importancia de la investigación sanitaria en humanos e identifica las implicaciones éticas y sociales involucradas en dicha investigación, tales como los beneficiarios de la investigación, el consentimiento, los múltiples valores culturales, las diferencias en la atención sanitaria en los países, la compatibilidad de los lineamientos éticos promulgados internacionalmente y las responsabilidades de los comités de ética tanto locales como extranjeros. ${ }^{9}$

Cuando se realizan investigaciones en países en desarrollo y están financiadas por países desarrollados, se plantean unas cuestiones éticas que tienen relevancia. Los países en desarrollo necesitan la investigación sanitaria para ayudar a combatir la elevada tasa de mortalidad. Para evitar que se presenten explotaciones en el marco de las investigaciones patrocinadas desde el exterior, dejando por fuera las prioridades nacionales, se proponen unos principios éticos que en lo personal creo que complementan los ya expuestos en el Informe Belmont y que sirven de marco para articular los deberes, las obligaciones, las reclamaciones y las expectativas de quienes participan en la investigación:

- Deber de mostrar respeto hacia las personas.

- Deber de aliviar el sufrimiento.

- Deber de ser sensibles a las diferencias culturales.

- Deber de no aprovecharse de la vulnerabilidad.

Además de las consideraciones éticas para la elaboración de un protocolo de investigación, se debe tener en cuenta en el caso de los grupos controles, el adecuado nivel de asistencia,"si procede, se debe ofrecer a los participantes del grupo testigo un nivel de asistencia universal para la enfermedad objeto de estudio. Cuando ello no sea oportuno, el nivel de asistencia que se debe ofrecer como mínimo al grupo testigo es la mejor intervención disponible en ese momento como parte del sistema nacional de salud pública." (Entendiendo como asistencia universal el mejor tratamiento disponible en cualquier parte del mundo para una enfermedad o afección determinada). 
La aplicación de un sistema de asistencia más bajo, no solo supondría aprovecharse de la vulnerabilidad de los participantes sino también cometer un error adicional al perpetuar una injusticia. En caso que esto no sea posible por las condiciones del país anfitrión, se debe evaluar de manera crítica las circunstancias en las cuales se realizará la investigación para establecer si las variaciones en las circunstancias proporcionan o no una razón pertinente desde el punto de vista moral, para ofrecer un nivel de asistencia diferente, para que no se cometan injusticias en dichos países y que nunca sea menor al que el país trata de proporcionar a nivel nacional.

El Consejo llega a la conclusión de que no es aceptable comenzar ninguna investigación sin haber adoptado una decisión acerca de si al concluir el ensayo se ofrecerá una intervención que ha resultado un éxito a quienes participaron en el grupo testigo. Los investigadores se deben comprometer a garantizar que tras su conclusión tendrán acceso a intervenciones eficaces todos los participantes en el ensayo que puedan beneficiarse de ellas durante un tiempo determinado. Además, antes de realizar la investigación se debería examinar la posibilidad de introducir y mantener un tratamiento eficaz para una comunidad más amplia, incluyendo esta información en el consentimiento informado.

Concluyendo, "la flexibilidad para ajustar los niveles de asistencia y los procedimientos de obtención del consentimiento para proyectos de investigación específicos debe ir unida a la preparación de un proceso riguroso y eficaz de examen ético que evalúe la idoneidad de la investigación propuesta. Esto permitirá formular la investigación de manera que tenga la máxima probabilidad de proporcionar información pertinente acerca de una población y de esta manera aliviar el sufrimiento sin correr el riesgo de explotar a las comunidades vulnerables."

En lo referente al tratamiento en el grupo control así como a los beneficios a que tendrán derecho los participantes al finalizar las investigaciones, la controversia no está terminada, ya que en caso de no hacer una revisión muy cuidadosa de los protocolos por parte de los Comités de Ética de Investigación(CEI) capacitados, se abre la posibilidad de cometer injusticias en los países anfitriones al no ofrecerles los mejores tratamientos disponibles o la disponibilidad de tratamientos eficaces al final de la investigación, estableciendo como lo expuse arriba un doble estándar ético.

Después de este panorama histórico de lo que ha sido la investigación con seres humanos y ante la evidencia de las experiencias nefastas cometidas que dieron origen a numerosas legislaciones internacionales y que a pesar de ellas seguimos siendo testigos, en la actualidad, de arbitrariedades cometidas principalmente en países en desarrollo, vuelvo a la pregunta inicial de este artículo, ¿Es entonces correcto investigar con seres humanos?

Para la autora Maria Luisa Pfeiffer cuando un sujeto es utilizado en una investigación se convierte en objeto de experimentación, en medio para conseguir un fin, ya sea éste el conocimiento, la tecnología o la ganancia monetaria. Adquiere la condición de objeto de experimentación y pierde su carácter de sujeto. Si se consideran los seres humanos libres e iguales no podemos someterlos a ningún tipo de violencia y manipulación por parte de otros seres humanos. Agrega la autora que "la persona tiene valor absoluto en sí misma, es depositaria de una dignidad, es decir de un respeto por sí misma que exige de los demás. Los seres humanos, las personas, son fines, no pueden ser usados como medios, de modo que si pensamos a los humanos como sujetos de derecho, como seres con dignidad es decir dignos de respeto, merecedores de reconocimiento por el simple hecho de ser humanos, libres y autónomos no podríamos experimentar con ellos"10

¿Cómo resolver lo anterior? Teniendo en cuenta que es necesario obtener conocimientos para aplicarlos a los hombres y más en el campo de la medicina donde se deben validar las nuevas técnicas de diagnóstico y tratamiento para mejorar la salud y la calidad de vida de todos. La autora sugiere entonces, que basta con cambiar la forma de leer la preposición "con" en 
la frase "investigación con seres humanos" dándole un sentido diferente, al entenderla como "compañía, en conjunto" y así de forma casi inmediata el sujeto recupera su papel protagónico y se convierte en parte de la investigación. ${ }^{10}$

Siendo así, el sujeto es capaz de participar en forma activa en el proceso de obtención del consentimiento informado, exigiendo toda la información clara, pertinente, completa y precisa para poder consentir de forma autónoma y voluntaria, exigiendo además que los beneficios de la investigación estén a su alcance si llegan a ser beneficiosos lo mismo que las compensaciones que sean necesarias, que se mantenga la confidencialidad, pero sobre todo, podrá preocuparse por determinar si la investigación responde a las necesidades de la comunidad, podrá estar pendiente de que sea beneficiosa y de que se publiquen los hallazgos tanto positivos como negativos.

Teniendo en cuenta entonces al sujeto como parte protagónica de la investigación, esta deberá realizarse bajo condiciones estrictas de respeto para quien participa así como de cuidado, ya que aumenta sus condiciones de vulnerabilidad al exponerse a unos riesgos no siempre conocidos y muchas veces sin beneficio directo para su persona. Este respeto se refleja no sólo en el correcto proceso de obtención del consentimiento informado sino en la elaboración de un protocolo de investigación con metodología correcta, que siga todas las normas necesarias, responda de la manera más adecuada a la pregunta de investigación, sirva a las necesidades de los sujetos o de sus comunidades, tenga un adecuado balance de los riesgos y beneficios así como la selección equitativa, justa y en número adecuado de los participantes.

Los investigadores tienen una doble responsabilidad ante lo arriba expuesto: así como deben diseñar las investigaciones correctas desde el punto de vista metodológico para obtener los conocimientos de nuevas intervenciones que permitan el avance de la ciencia, tienen que velar por la protección de los sujetos que participan, por sus derechos como seres humanos y por su bienestar durante la investigación.
Esta es una meta ideal que debería cumplirse en todas las investigaciones que involucran seres humanos y por difícil que parezca, todos los involucrados, científicos, patrocinadores, gobernantes deben comprometerse a llevarla a cabo, contando con la ayuda de un ente designado en forma específica para velar por lo anterior: los comités de ética en investigación con seres humanos (CEI).

Los avances científicos y tecnológicos que implican la investigación con seres humanos, así como las amargas experiencias históricas en donde se cometieron abusos en este campo, son el marco de referencia para el surgimiento y desarrollo de la ética de investigación con seres humanos y de los comités de ética. Estos nos permiten evaluar "un proyecto de investigación en su calidad ética y científica mediante la adquisición de hábitos que nos sensibilicen con relación al respeto de los derechos de los sujetos de investigación y que nos haga receptivos al espíritu de las normas y códigos internacionales de ética de investigación en humanos." ${ }^{\prime 1}$

Los principales lineamientos de los comités de ética de la investigación, la conformación, objetivos, forma de operar y requisitos básicos para presentar trabajos de investigación están definidos en numerosas publicaciones: "El propósito de un comité de Ética de Investigación como ente multidisciplinario independiente es asegurar la protección de los derechos, dignidad, seguridad y bienestar de los sujetos humanos participantes en investigación."12

De acuerdo con las Guías Operacionales para Comités de Ética que evalúan Investigación Biomédica, de la Organización Mundial de la Salud (OMS) de Ginebra publicadas en el año 2002,"los estándares éticos y científicos para llevar a cabo investigación biomédica en humanos han sido desarrollados y establecidos en guías internacionales, incluyendo la Declaración de Helsinki, las Guías Éticas Internacionales para Investigación Biomédica que Involucra a Humanos, del CIOMS y de la OMS, y las Guías para Buena Práctica Clínica, del ICH, publicadas en 1996. El cumplimiento de estas guías ayuda a asegurar que se promuevan la dignidad, 
derechos, seguridad y bienestar de los participantes en la investigación, y que los resultados de las investigaciones sean creíbles.

Todas las guías internacionales requieren de la revisión ética y científica de la investigación biomédica, así como del consentimiento informado y de la protección apropiada de aquellos incapaces de consentir, como medidas esenciales para proteger al individuo y a las comunidades que participan en la investigación. Para los propósitos de estas guías, la investigación biomédica incluye investigación farmacéutica, equipo médico, radiación médica e imagen, procedimientos quirúrgicos, historias clínicas y muestras biológicas, así como investigaciones epidemiológicas, sociales y psicológicas.

Estas publicaciones intentan facilitar y apoyar la revisión ética en todos los países del mundo. Están basadas en un examen minucioso de los requerimientos para revisión ética, como lo establecen las guías internacionales, así como en una evaluación de las prácticas existentes de revisión ética en todo el mundo. Sin embargo, no intentan sustituir la necesidad de guías nacionales y locales para la evaluación ética de la investigación biomédica, ni intentan reemplazar leyes y reglamentos nacionales. ${ }^{13}$

Para concluir, estos comités (CEI) tienen como objetivo contribuir al desarrollo de calidad y consistencia en la evaluación ética de investigación biomédica, constituyen su parte primordial, y brindan protección a los pacientes que son sujetos de investigación. El comité es un grupo multidisciplinario independiente, cuya responsabilidad es asegurar la protección de los derechos, dignidad, seguridad y bienestar de los seres humanos sujetos de investigación y debe actuar en interés total de los participantes de la investigación y de las comunidades, tomando en consideración las leyes e instituciones regulatorias donde se lleva a cabo la investigación.

Termino respondiendo afirmativamente a la pregunta planteada al inicio de este artículo: en la medida que durante una investigación, los sujetos actúen como parte de la misma, con un papel protagónico, siendo sujetos y no "objetos" de experimentación, en la cual respetemos su dignidad como persona humana y por consiguiente sus derechos, es ético realizar investigaciones con seres humanos dentro de un estricto marco normativo y con la adecuada supervisión de los CEI.

Al defender al sujeto como ser autónomo, digno, protegiendo su vida, su salud y su intimidad, por ser merecedor de reconocimiento y libre, se convierte en protagonista de la investigación. Es ahora, más que nunca, cuando adquiere toda la relevancia el imperativo propuesto por Kant en 1785:“obra siempre de tal modo que uses a la humanidad, tanto en tu persona como en la persona de cualquier otro, al mismo tiempo como fin y nunca sólo como medio"14.

\section{Referencias}

1. Gracia D. Investigación Clínica. Investigación y justicia sanitaria. Profesión Médica. En: Red Latinoamericana de Bioética. UNESCO. Primer curso de ética de investigación con seres humanos. Programa de educación permanente a distancia; 2006.

2. Brussino SL. Antecedentes y fundamentos de la ética de la investigación. En: Red Latinoamericana de Bioética. UNESCO. Primer curso de ética de investigación con seres humanos. Programa de educación permanente a distancia; 2006.

3. Freedman B. Human research: unethical research. En: Warren TR, editor. Encyclopaedia of bioethics. Ed. rev. New York: The Free Press; Simon and Schuster Mac Millan; 1995.

4. Comisión Nacional para la Protección de los Sujetos Humanos de Investigación Biomédica y del Comportamiento. Informe Belmont: principios y guías éticos para la protección de los sujetos humanos de investigación. [Washington]: Comisión Nacional para la Protección de los Sujetos Humanos de Investigación Biomédica y del Comportamiento; 1979.

5. Consejo de las Organizaciones Internacionales de Ciencias Médicas, Organización Mundial de la Salud. Guías éticas internacionales para la investigación biomédica que involucra seres humanos. Chile : OPS, CIOMS; 2002.

6. Colombia. Ministerio de Salud. Normas científicas, técnicas y administrativas para la investigación en salud: Resolución 8430. Bogotá: Ministerio de Salud; 2006.

7. Kottow M. Bioética frente a ciencia y técnica. En: Introducción a la bioética. 2a ed. Santiago de Chile: Editorial Mediterráneo; 2005. p. 145-58.

8. Kottow M. Modelos de evaluación y situaciones especiales. En: Red Latinoamericana de Bioética. UNESCO. Primer curso de ética de investigación con seres humanos. Programa de educación permanente a distancia; 2006. 
9. Nuffield Council on Bioethics. Ética de la investigación relativa a la atención sanitaria en los países en desarrollo. El resumen, las conclusiones y las recomendaciones contenidas en las normas CIOMS. [Sitio en Internet]. [citado 10 Oct. 2006]; [aprox. 3 p.]. Disponible en: http://www. puc.cl/enfermeria/html/noticias/doc/cioms.pdf

10. Pfeiffer ML. ¿Es ético experimentar con humanos? En: Red Latinoamericana de Bioética. UNESCO. Primer curso de ética de investigación con seres humanos. Programa de educación permanente a distancia; 2006.

11. Estevez Montalban A, Bota i Arqué A, Fernández Milla L, Hernández M, Hevia Larenas A, Lara Alvarez C. Los comités de evaluación ética y científica de la investigación en seres humanos en los países latinoamericanos y El Caribe: una propuesta para su desarrollo Centro Interdisciplinario de Estudios en Bioética (CIEB) de la Universidad de Chile; 2004.

12. AVANZAR. ABC en la construcción y funcionamiento de los comités de ética en investigación. Bogota; 2003.

13. Organización Mundial de la Salud. Guías operacionales para comités de ética que evalúan investigación biomédica. Ginebra: OPS; 2002.

14. Kant I. Fundamentación para una metafísica de las costumbres. Filosofía. Madrid: Alianza; 2002.
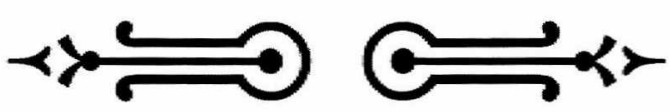